\title{
GAUGE INVARIANT INTERACTIONS OF CLASSICAL FIELDS
}

\author{
P. K. SMRZ \\ (Received 19 March 1973) \\ Communicated by C. A. Hurst.
}

\begin{abstract}
The theory of Yang-Mills fields is explicitly formulated in terms of the theory of connections in principal and associated fiber bundles. Special attention is paid to the fiber bundles with Lorentz and Poincaré structures. Equations of the form $\nabla \psi=0$, where $\nabla$ is a generalized convariant derivative, are shown to contain "mass" terms if the connection in the Poincaré fiber bundle is cononically associated to the connection in the Lorentz fiber bundle.
\end{abstract}

\section{Introduction}

The concept of gauge invariant interactions (or Yang-Mills fields) is an attractive way to unify the theory of interacting physical fields (Yang and Mills (1954), Utigama (1956)). It is known that the theory of Yang-Mills fields is parallel to the theory of connections in vector bundles (Hermann (1970)). Geometrical meaning of the gauge invariance of the theory becomes even more transparent when the formalism of principla and associated fiber bundles is uded. The explicit correspondence of differential geometric and physical terms is described in section 1. In section 2 fiber bundles with the Lorentz group structure are discussed. The covariance derivative is generalized for the case when the structure group acts upon the tangent vector space of the base manifold. Solutions of the Dirac equation with zero mass are shown to be horizontal cross sections in the generalized sense. In Section 3 the structure group is enlarged to the Poincare group. The equations satisfied by the horizontal cross sections acquire additional terms that are of simple form (mass-like) if the connection in such an enlarged fiber bundle is canonically associated with the connection in the original fiber bundle.

\section{Gauge invariance and fiber bundles}

Let $F$ be a linear vector space and $\lambda$ a function* from a manifold $M$ to $F$ defined on an open subset of $M$. Such a function will describe a physical field

* In this paper we do not deal with questions of differentiability. Every function is considered to be as many times differentiable as needed in explicit statements. 
while $M$ will be the space-time manifold. Descriptions of physical fields are usually invariant with respect to gauge transformations in the following sense: There is a Lie group $G$ acting differentiably on $F$ such that for every function $x \rightarrow g(x) \in G$, $x \in M$, defined on the same subset of $M$ as $\psi, g(x) \psi(x)$ describes a field physically equivalent to $\psi(x)$. To describe such a situation mathematically, we must look for a structure where one element corresponds to the whole class of fields $g(x) \psi(x)$ with different $g(x)$ 's. Such a structure is the family of cross sections in an associated fiber bundle, as explained further.

Let $P(G, M)$ be a principal fiber bundle (Kobayashi and Nomizu (1963)) $P$ is a manifold on which Lie group $G$ acts differentiably without fixed point (on the right, say). $M$ is the quotient space of $P$ by the equivalence relation induced by $G$. Orbits of $G$ in $P$ are called fibers. We now form $P \times F$ and define action of $G$ on it by

$$
g(p, \psi)=\left(p g, g^{-1} \psi\right), g \in G, p \in P, \psi \in F .
$$

Define $E$ as the quotient space $(P \times F)_{G}$ with respect to such action of $G$ on $P \times F$. Then a point $(p, \psi)_{G} \in E$ is identical with $\left(p g, g^{-1} \psi\right)_{G} \in E$ for all $g \in G$.

A map $\phi: M \rightarrow E$ contains the gauge invariance as

$$
\phi(x)=(p(x), \psi(x))_{G}=\left(p(x) g^{-1}(x), g(x) \psi(x)\right)_{G}
$$

$E$ is called a fiber bundle associated to $P$ with standard fiber $F . \phi(x)$, a cross section in $E$, is what corresponds to a given physical state of field $\psi$. Choice of a particular function $\psi$ in one of the equivalent classes is determined by a corresponding $p(x)$, a cross section in $P$.

An alternative way of describing $\phi(x)$ is by an $F$-valued function on $P$ : If $p_{0}$ is any point on the same fiber as $p(x)$ we define a function $\xi: P \rightarrow F$ as follows

$$
(p(x) \psi(x))_{G}=\left(p_{0}, \xi\left(p_{0}\right)\right)_{G} .
$$

This defines $\xi$ at all points of $P$ that project onto a subset of $M$ on which $\psi$ is defined. Dependence of $\xi(p)$ on $p$ when it moves along a fixed fiber is trivial:

so that

$$
(p, \xi(p))_{G}=(p g, \xi(p g))_{G}=(p, g \xi(p g))_{G}
$$

$$
\xi(p g)=g^{-1} \xi(p) .
$$

There is a one-one correspondence between the set of functions $\xi$ and the set of cross sections in the associated fiber bundle. A physical field $\psi(x)$ may be recovered from $\xi$ by choosing a cross section $p(x)$ in the principal fiber bundle $P$ :

$$
\psi(x)=\xi(p(x)) \text {. }
$$

Functions $\psi$ satisfy differential equations characteristic for the physical fields they describe. Such equations involve directional derivatives $X \psi(x), X$ being a tangent vector at $x \in M$. 
Equations describing behaviour of a free (i.e., non-interacting) field $\psi(x)$ are normally invariant with respect to a fixed gauge transformation

$$
\psi(x) \rightarrow g \psi(x), g \in G .
$$

The same equations will not be invariant with respect to a general gauge transformation $\psi(x) \rightarrow g(x) \psi(x)$ because of the effect of differentiation on $g(x)$. A covariant derivative $\nabla_{X}$ is then sought with the property

$$
\nabla_{X} g(x) \psi(x)=g(x) \nabla_{x} \psi(x)
$$

It means that in the fiber bundle formalism we need a directional derivative that is not affected by changes that occur along the fibers. If the fibers are thought of as indicators of vertical direction, all we need is to specify a horizontal direction. A covariant derivative should then be connected with a horizontal directional derivative.

In differential geometry, a horizontal direction is specified by a connection in the fiber bundle. Let $\Gamma$ be a connection in $P$. Then if $X_{p}$ is a tangent vector at $p \in P$ we have a unique decomposition

$$
X_{p}=Y_{p}+Z_{p}
$$

where $Y_{p}$ is horizontal, and $Z_{p}$ vertical. The connection $\Gamma$ may be also characterized by the connection form $\omega$, which is a differential 1 -form on $P$ with values in the Lie algebra $\mathscr{G}$ of the structure group $G$, satisfying

(1) $\omega\left(X_{p}\right)=0$ if $X_{p}$ is horizontal,

(2) $\omega\left(X_{p}\right)=A$ if $X_{p}$ is vertical, $A \in \mathscr{G}$ corresponding to the vector $X_{p}$ in the following way: Let $G_{p}$ be a subspace of the tangent vector space at $p \in P$ which is naturally isomorphic to the tangent vector space of group $G$ at the identity via the action of $G$ on $P$. The isomorphism of the latter with $\mathscr{G}$ provides the correspondence.

Let us now return to the problem of covariant derivative. Function $\phi$ or $\xi$ is considered to be given, while a pair of $p(x)$ and $\psi(x)$ is chosen in the equivalence class. If $X$ is a tangent vector at $x \in M, X_{p}$ will denote a tangent vector at $p(x)$ such that it is tangent to the cross section $p$ considered as a submanifold of $P$, and its natural projection is $X$. The covariant derivative $\nabla_{X}$ is then defined by

$$
\nabla_{X} \psi(x)=Y_{p} \xi(p) /_{p=p(x)},
$$

where $Y_{p}$ is the horizontal component of $X_{p}$. It is easy to show that it satisfies property (8) (see Smrz (1973a)). Rewriting $Y_{p}$ as $X_{p}-Z_{p}$ and using

as well as

$$
\left.X_{p} \xi(p)\right|_{p=p(x)}=X \psi(x)
$$

$$
Z_{p} \xi(p) /_{p=p(x)}=-\omega\left(X_{p}\right) \xi(p(x))^{*}
$$

* Vector space $F$ is considered as a representation space of Lie algebra $\mathscr{G}$, and $\omega\left(X_{p}\right)$ is the corresponding matrix. 
we can write the covariant derivative as

$$
\nabla_{X} \psi(x)=X \psi(x)+\omega\left(X_{p}\right) \psi(x)
$$

If $X=\partial / \partial x^{\mu}$, the last expression may be written more explicitly

$$
\nabla_{\mu} \psi(x)=\frac{\partial \psi(x)}{\partial x^{\mu}}+\sum_{a=1}^{n} B_{\mu}^{a}(x) A_{a} \psi(x),
$$

where $\nabla_{\mu}$ denotes the covariant derivative with respect to $\partial / \partial x^{\mu}, A_{a}, a=1, \cdots, n$, form a basis of Lie algebra $\mathscr{G}$, and $B_{\mu}^{a}(x)$ are components of the connection form $\omega$.

$B_{\mu}^{a}(x)$ (or, in fact, rather $-B_{\mu}^{a}(x)$ in literature) are the Yang-Mills fields interacting with the system described by $\psi(x)$ and $p(x)$.

\section{Fiber bundles with Lorentz structure}

If the structure group of the principal fiber bundle acts not only on manifold $P$ and vector space $F$, but also on the tangent vector space $M_{x}$ at every point $x$ of the base manifold $M$, the concept of the covariant derivative may be naturally generalized. Besides satisfying (8) for every given vector $X$ we may try to find a tangent vector $X_{0}$ such that

$$
\nabla_{g(x) X_{0}} g(x) \psi(x)=g(x) \nabla_{X_{0}} \psi(x) .
$$

If $X_{0}=\sum_{\mu=1}^{m} a^{\mu} \partial / \partial x^{\mu}$ it means that

$$
\sum_{\mu=1}^{m} a^{\mu^{\prime}} \nabla_{\mu} g(x) \psi(x)=\sum_{\mu=1}^{m} a^{\mu^{\prime}} g(x) \nabla_{\mu} \psi(x)=g(x) \sum_{\mu=1}^{m} a^{\mu} \nabla_{\mu} \psi(x)
$$

where

$a^{\mu^{\prime}}=\sum_{v=1}^{m} D_{v}^{\mu}(x) a^{\nu}, D_{v}^{\mu}(x)$ representing the action of the group element $g(x)$ on the tangent vector space at $x \in M$ if the basis $\left\{\partial / \partial x^{\prime}, \cdots, \partial / \partial x^{m}\right\}$ is used. Condition (12) will be true for all functions $\psi(x)$ only if

$$
a^{\mu^{\prime}}=g(x) a^{\mu} g^{-1}(x) \text {. }
$$

Of course, if $a^{\mu}$ are real numbers, and $G$ acts on $M_{x}$ with only the null vector being fixed, there is no non-trivial solution for (13).

On the other hand, a solution may exist, if coefficients $a^{\mu}$ are allowed to be matrices of the same order and over the same field as those representing elements of group $G$ and its Lie algebra $\mathscr{G}$. Also, the linear properties of the connection form $\omega$ may be extended to

$$
\omega(a X+b Y)=a \omega(X)+b \omega(Y)
$$

with $a$ and $b$ being matrices. Note that $\omega(X)$ itself is a matrix of the same type, and the multiplication is well defined. Then 


$$
\nabla_{\Sigma_{\mu=1}^{m}}^{m} a^{\mu} X_{\mu}=\sum_{\mu=1}^{m} a^{\mu} \nabla_{X_{\mu}}
$$

just as for the real coefficients.

As an example we consider $G$ to be the homogeneous Lorentz group. We assume further that $M$ is a four-dimensional manifold such that at each point $x \in M$ there exists a basis

$$
h:\left\{\sum_{\mu=1}^{4} h_{k}^{\mu}(x) \frac{\partial}{\partial x} \mu, \quad k=1,2,3,4\right\}
$$

with the following properties: If $L_{l}^{k}(g)$ is the four-dimensional real representation of $g \in G$ satisfying the usual orthogonality relations

$$
\begin{aligned}
& \sum_{l_{1} n=1}^{4} L_{k}^{l}(g) g_{l n} L_{m}^{n}(g)=g_{k m}, \quad g \in G, \\
& g_{l n}=0 \text { if } l \neq n, \quad g_{11}=g_{22}=g_{33}=-g_{44}=1 \text {, }
\end{aligned}
$$

then

$$
\sum_{l=1}^{4} L_{l}^{k}(g) a^{l}=a^{k^{\prime}}
$$

represents the action of $G$ on the tangent vector space of point $x$ if $h$ is used as the basis. In order to solve condition (13) we have to find $a_{l}, l=1,2,3,4$, satisfying

$$
g a^{l} g^{-1}=\sum_{k=1}^{4} L_{k}^{l}(g) a^{k}
$$

If $F$ is the four-dimensional complex vector space and the generators of the Lorentz group are written in the form $S_{k l}=\frac{1}{4}\left(\gamma^{k} \gamma^{l}-\gamma^{l} \gamma^{k}\right)$, where $\gamma_{k}, k=1,2,3,4$, are the Dirac matrices satisfying $\gamma^{k} \gamma^{l}+\gamma^{l} \gamma^{k}=2 g_{k l} I$, then matrices $a^{k}$ may be chosen as proportional to $\gamma^{k}$,

$$
a^{k}=c \gamma^{k}, \quad c \in \mathbb{C} .
$$

The coefficients $a^{\mu}$ related to basis $\left\{\partial / \partial x^{\prime}, \cdots, \partial / \partial x^{4}\right\}$ are then obtained by $a^{\mu}=\sum_{k=1}^{4} h_{k}^{\mu}(x) a^{k}$. The operator $\nabla_{X_{0}}=\nabla$ is what we call the generalized covariant derivative. The equation

$$
\nabla \psi(x)=0
$$

is identical to the Dirac equation without the mass term. It includes the Yang-Mills fields $B_{\mu}^{a}(x), a=1, \cdots, 6$, describing the field of gravitation. Both $B_{\mu}^{a}(x)$ and $h_{k}^{\mu}(x)$ are, of course, connected with the Riemannian metric on $M$. A relation connecting $B_{\mu}^{a}(x)$ and $h_{k}^{\mu}(x)$ was derived in Utiyama (1956) under certain simplifying assumptions. A more general and simple derivation based on the fiber bundle formalism is given in Smrz (1973a). 


\section{Extension to Poincaré structure.}

It is possible to introduce a mass term into the equation (14) without loosing its homogeneity. In fact, the mass term comes from a set of additional Yang-Mills fields not included in considerations of section 2 . The only way to bring more Yang-Mills fields into the formalism is to enlarge the structure group of the principal fiber bundle. We shall consider now a principal fiber bundle with the Poincaré group $\widetilde{G}$ as its structure group. $\widetilde{G}$ has a form of a semidirect product $\widetilde{G}=V \times G$, where $G$ is the homogeneous Lorentz group, and $V$ is the fourdimensional real vector space isomorphic to the abelian group of translations. Similarly the Lie algebra $\tilde{\mathscr{G}}$ of group $\tilde{G}$ has a form of a semi-direct sum $\tilde{\mathscr{G}}=V+\mathscr{G}$. If $\widetilde{P}$ is a product manifold $V \times P$ with $P$ being the manifold of Lorentz frames as defined in the previous section, then every connection form $\tilde{\omega}$ in $\widetilde{P}(\widetilde{G}, M)$ uniquely decomposes according to

$$
i^{*} \tilde{\omega}=\omega+\alpha,
$$

where $i$ is the natural injection of $P$ into $\widetilde{P}\left(i^{*}\right.$ denotes the mapping of differential forms induced by the manifold map $i$ ), $\omega$ is a $\mathscr{G}$-valued 1 -form on $P$, and $\alpha$ is a $V$-valued 1 -form on $P$. In the covariant derivative $(10) \alpha$ will generate additional four fields $B_{\mu}^{a}(x)$. These fields are connected with the group of translations, but as long as these vertical translations along fibers of $\widetilde{P}$ are not somehow related to the "real" translations in the base manifold, their form is not specified any further. On the other hand, there is a natural way of defining such a relationship, known from studies of the affine connections. We shall briefly review the construction as it applies to the structures we study.

Every point $p$ of the bundle of Lorentz frames $P$ may be considered as a linear isomorphism $V \rightarrow M_{x}$. Quantities $h_{k}^{\mu}, \mu, k=1,2,3,4$, that characterize a point in $P$ are an explicit matrix formulation of such isomorphism. A 1-form with values in $V$ may be defined on $P$ by

$$
\theta_{p}\left(X_{p}\right)=p^{-1} \pi\left(X_{p}\right)
$$

where $X_{p}$ is a tangent vector at $p \in P, \pi$ is the projection map so that $\pi\left(X_{p}\right)$ is a tangent vector at $x=\pi(p) \in M$. Differential form $\theta$ has the same properties as $\alpha$ with respect to the action of group $G$, and it is possible to set

$$
\theta=\alpha \text {. }
$$

A connection in $\widetilde{P}(\widetilde{G}, M)$ that satisfies $(16)$ is in the theory of affine connections called the connection canonically associated with $\omega$ in $P(G, M)$. In the explicit form with fixed bases in $V$ and $M_{x}$ the condition (16) reads

$$
B_{\mu}^{k}(x)=h_{\mu}^{k}(x)
$$

where $B_{\mu}^{k}(x)$ is the Yang-Mills field associated with the translation in the direction 
of $k$ th axis, and $h_{\mu}^{k}$ is the usual (a little confusing) notation for the inverse of matrix $h_{k}^{\mu}$.

Let us now return to the equation $\nabla \psi=0$ with $\nabla$ being the generalized covariant derivative. We shall write it in an explicit form

$$
\sum_{\mu=1}^{4} \sum_{k=1}^{4} h_{k}^{\mu}(x) a^{k}\left(\frac{\partial}{\partial x^{l}}+\sum_{l=1}^{4} B_{\mu}^{l}(x) p_{l}+\sum_{a=5}^{10} B_{\mu}^{a}(x) A_{a}\right) \psi=0,
$$

where $A_{a}, a=5, \cdots, 10$, are the generators of the Lorentz group, while $p_{l}$, $l=1, \cdots, 4$, generate translations. Using (17) the terms connected with translations simplify as follows:

$$
\sum_{\mu=1}^{4} \sum_{k=1}^{4} \sum_{l=1}^{4} h_{k}^{\mu}(x) a^{k} B_{\mu}^{l}(x) p_{l}=\sum_{k=1}^{4} a^{k} p_{k}
$$

This is a constant matrix that, due to the properties of matrices $a_{k}$ and $p_{k}$, commutes with all elements of the homogeneous Lorentz group. It is not necessarily a multiple of the identity matrix, and its form will depend on a particular representation of the Poincaré group as well as on the choice of matrices $a^{k}$. The mass term of the Dirac equation is obtained in the correct form if $a^{k}$ is proportional to $\gamma^{k}$, and $p_{k}$ is chosen as $1 / R \gamma^{k}$, where the Poincaré group is considered a contracted (in Wigner's sense, Inönü and Wigner (1953)) de Sitter group.

Finally, we shall consider a weaker condition than (16). The translation part $\alpha$ of the connection form may still be in a relationship to the canonical form $\theta$, but

$$
\alpha\left(X_{p}\right)=\theta\left(\phi_{p} X_{p}\right)
$$

where $\phi_{p}$ is an automorphism of the tangent vector space at $p$. If $R_{g}$ denotes the action of $g \in G$ on $P$

$$
\alpha_{p g}\left(R_{g} X_{p}\right)=R_{g^{*}} \alpha_{p}\left(X_{p}\right)=R_{g^{*}} \theta_{p}\left(\phi_{p} X_{p}\right)=\theta_{p g}\left(R_{g} \phi_{p} X_{p}\right)
$$

but also

$$
\alpha_{p g}\left(R_{g} X_{p}\right)=\theta\left(\phi_{p g} R_{g} X_{p}\right)
$$

so that the relation (19) makes sense only if

$$
\phi_{p g}=R_{g} \phi_{p} R_{g-1} \text {. }
$$

In particular, if $\phi_{p}$ defines an automorphism $\phi_{x}: M_{x} \rightarrow M_{x}$ by

$$
\phi_{p}\left(X_{p}\right)=\phi_{x}\left(\pi\left(X_{p}\right)\right), \quad x=\pi(p),
$$

then $\phi_{x}$ must commute with $R_{g}$. But $G$ (Lorentz group) acts irreducibly on $M_{x}$, therefore the only automorphism commuting with $R_{g}$ for all $g \in G$ is a multiple of identity. In such a way we obtain a condition

$$
\alpha_{p}=m(x) \theta_{p}, \quad x=\pi(p) .
$$


The mass terms obtained while condition (21) holds differ from the canonical terms of condition (16) only by a scalar function on $M$.

\section{Conclusions}

We can say that a physical system of a particle interacting with a system of classical fields corresponds to the geometrical system of a principal fiber bundle with connection plus an associated fiber bundle. The connection contains all information about the system of classical fields, while the wave function of the particle corresponds to a cross section in the associated fiber bundle. In case of a Dirac particle the cross section is horizontal in a generalized sense. A new relationship between translations and the mass of the particle is suggested by such an approach. There are many questions that remain to be answered. It is, for example, the question of dynamical equations for the classical fields. What is the form of a general dynamical equation for a connection in a principalfiber bundle? What is its relation to the usual equations for the field of gravitation? Finally, the formalism should be applicable also to the system of interacting quantum fields. Unfortunately, the structure group of the fiber bundle must be non-compact if it is to contain the Lorentz group, and this is expected to bring many difficulties into quantized theory.

\section{Acknowledgement}

The author is grateful to Mr. L. Kavalieris for numerous fruitful discussions.

\section{References}

R. Hermann (1970), Vector Bundles in Mathematical Physics, (W. A. Benjamin, New York 1970).

E. Inonu and E. P. Wigner (1953), 'On the contraction of groups and their representations', Proc. Nat. Acad. Sci. U.S. 39, 510.

S. Kobayashi and K. Nomizu (1963), Foundations of differential geometry, Vol. I, (Interscience Pub. 1963).

P. Smrz (1973), 'Fibre bundles and Yank-Mills fields', J. Austral. Math. Soc. 15, 482.

P. Smrz (1973a), 'Gauge invariance of the Dirac equation', J. Austral. Math. Soc. 15, 117.

R. Utiyama (1956), 'Invariant theoretical interpretation of interaction', Phys. Rev. 101, 1597.

C. N. Yang and R. N. Mills (1954), 'Conservation of isotopic spin and isotopic gauge invariance', Phys. Rev. 96, 191.

Department of Mathematics

University of Newcastle

N. S. W. 2308

Australia 